\title{
Microbiological Efficacy of Photodynamic Therapy as an Adjunct to Non-surgical Periodontal Treatment: A Clinical Trial
}

\author{
Mohammadreza Talebi ${ }^{1,2}$, Rojin Taliee ${ }^{2^{*}}$, Masoud Mojahedi ${ }^{3}$, Mansour Meymandi ${ }^{2}$, Maryam Torshabi ${ }^{4}$ \\ ${ }^{1}$ Dental Research Center, Research Institute of Dental Sciences, Shahid Beheshti University of Medical Sciences, Tehran, Iran \\ ${ }^{2}$ Department of Periodontics, Shahid Beheshti University of Medical Sciences, Tehran, Iran \\ ${ }^{3}$ Laser Department of Periodontics, Shahid Beheshti University of Medical Sciences, Tehran, Iran \\ ${ }^{4}$ Department of Dental Biomaterials, Shahid Beheshti University of Medical Sciences, Tehran, Iran
}

\section{*Correspondence to \\ Rojin Taliee, DDS; Department \\ of Periodontics, Shahid Beheshti \\ University of Medical Sciences, \\ Tehran, Iran. \\ Tel: +98-9126792800; \\ Fax: +98-21-22711836 \\ E-mail: rojintaliee@gmail.com}

Published online 27 March 2016

\begin{abstract}
Introduction: The efficiency of routine scaling and root planning is negatively influenced by the tooth anatomy and residual bacteria all possibly affecting the treatment outcomes in future. The present study compared the microbiologic effectiveness of the photodynamic therapy (PDT) as an adjunctive treatment modality for nonsurgical treatment in chronic periodontitis.

Methods: In this randomized controlled clinical trial, 18 chronic periodontitis patients were selected. Four quadrants were randomly treated by scaling and root planning (SRP), diode laser (810n m wavelength, $1.5 \mathrm{~W}$ and $320 \mu \mathrm{m}$ fiber, contact and sweeping technique), SRP + PDT (with diode laser $808 \mathrm{~nm}, 0.5 \mathrm{~W}$ ) and laser + SRP (with diode laser $808 \mathrm{~nm}, 1 \mathrm{~W}$ ) in each patient. Presence of periodontal pathogen species in the treated areas were measured before the treatment, at 1 and 3 months afterwards. The identification and reproduction of the specific genes of pathogen bacteria were done by means of polymerase chain reaction (PCR) technique. Presence of oral pathogen bacteria in the treatment groups were analyzed by chi-square test. A semi quantitative analysis was used to measure the intensity of white light in each band. This was calculated by number of pixels in each band.

Results: In the qualitative analysis, Fusobacterium nucleatum (Fn) and Treponema denticola (Td) species were killed after 1 month in all treatment modalities. PDT had more effects to decrease Prevotella intermedia (Pi) species than SRP while Tannerella forsythensis count (Tf) species increased in all treatments. Furthermore, Actinobacillus actinomycetemcomitans (Aa) species decreased in all treatments and Porphyromonas gingivalis (P.g) species increased in all treatments after 1 and 3 months.

Conclusion: It can be concluded that PDT was more effective as an adjunctive treatment to SRP than SRP alone; however, no distinct differences were found between both treatment modalities regarding reduction of certain pathogen bacteria.

Keywords: Periodontitis, Chronic; Photodynamic therapy; Pathogen.
\end{abstract}

\section{Introduction}

Chronic periodontitis is an inflammatory response to accumulation of microbial plaque and calculus on teeth surfaces resulting in the destruction of tooth surrounding tissues. ${ }^{1}$ Periodontal therapy is performed aiming to remove dental biofilm from the root surfaces and cease the inflammatory process via decreasing the number of periodontopathogenic microorganisms. Success of periodontal therapy mainly depends on efficient removal of supragingival and subgingival microbial biofilm and smear layer, which contain bacteria, bacterial endotoxins and infected root cementum. ${ }^{2,3}$ Different types of lasers have been suggested as non-invasive techniques for treatment of chronic periodontitis to achieve more favorable therapeutic effects. ${ }^{4}$ Several laser systems with different wavelengths and exposure settings have also been used for periodontal therapy. Diode lasers are used for soft tissue treatments and despite their bactericidal efficacy, they are not effective for calculus removal from the root surfaces. Therefore, they may be helpful as an adjunct to scaling and root planning (SRP) due to having bactericidal and detoxing effects. ${ }^{5}$ Low-level laser has also been recommended for pain reduction and enhancing wound healing due to its anti-inflammatory effects. ${ }^{6}$ Low-level laser irradiation in conjunction with the use of a photosensitizer is also an efficient modality to reduce bacterial contamination of periodontal pockets. This method is known as antimicrobial photodynamic therapy (PDT). ${ }^{7,8}$

Please cite this article as follows: Talebi M, Taliee R, Mojahedi M, Meymandi M, Torshabi M. Microbiological efficacy of photodynamic therapy as an adjunct to non-surgical periodontal treatment: a clinical trial. J Lasers Med Sci. 2016;7(2):126-130. doi:10.15171/jlms.2016.21. 
PDT was first introduced by Raab in $1990^{\circ}$ and requires three components of light, photosensitizer and free radicals. ${ }^{10}$ Absence of genotoxic and mutagenic effects guarantees the long-term safety of this therapeutic method. This technique is beneficial for elimination of bacteria in a very short time. It does not damage the surrounding tissues, decreases the risk of bacteremia in immunocompromised patients with systemic conditions, decreases dentin hypersensitivity following root planning and prevents interference with the normal flora of the adjacent teeth. ${ }^{11,12}$ This clinical trial aimed to assess the microbiological effects of PDT as an adjunct to non-surgical periodontal therapy.

\section{Methods}

Sample Size and Method of Sampling

Analysis of qualitative variables required 14 patients in each intervention group compared to the control group. Considering the possible drop outs, $15 \%-20 \%$ increase in sample size was considered and sample size of 18 patients was used.

This clinical trial was approved by the ethics committee of Shahid Beheshti University, School of Dentistry and registered at IRCT.ir (registration ID: IRCT2015022221180N1). A total of 18 patients with chronic periodontitis presenting to the Periodontics Department of Shahid Beheshti University, School of Dentistry during 2011-2013 gave their written informed consent and were evaluated in this study. The inclusion criteria were confirmed diagnosis of moderate to severe chronic periodontitis, presence of at least 2 teeth with a pocket depth of $4-10 \mathrm{~mm}$ in each quadrant, gingival bleeding and presence of at least 5 natural teeth in each quadrant. The exclusion criteria were history of systemic diseases such as diabetes mellitus, cancer, AIDS, metabolic and endocrine diseases, pregnancy or nursing, chronic high-dose steroid therapy, history of previous or current radiotherapy, tobacco consumption, orthodontic therapy, antibiotictherapy in the past 6 months and periodontal therapy in the past 6 months. Microbiological sampling was performed at baseline, after one and three months. Based on the method of randomization, each patient quadrant was allocated one of four therapeutic modalities as follows: First modality: Conventional SRP alone (control group), Second modality: $\mathrm{SRP}+$ diode laser irradiation at a wavelength of $808 \mathrm{~nm}$ with $1.5 \mathrm{~W}$ power and $320 \mu \mathrm{m}$ fiber just after SRP (conventional technique for curettage), Third modality: SRP + PDT with $808 \mathrm{~nm}, 0.2 \mathrm{~W}$ power and $320 \mu \mathrm{m}$ fiber just after SRP and Fourth modality: Diode laser with $808 \mathrm{~nm}$, $1 \mathrm{~W}$ power for pocket decontamination followed by SRP after 48 hours. All laser treatments were done by Dr. Smile Diode Laser Device. Before the interventions, all patients received oral hygiene instructions and these instructions were repeated in the following sessions.

\section{Microbiological Analysis}

Samples were obtained from deepest pocket in each quadrant at baseline (before treatment), at 1 and 3 months after the treatment using sterile paper points. After sampling, each paper point (18 patients, 4 sites, 2 paper points for each site) was placed in $1.5 \mathrm{~mL}$ sterile microtubes containing $200 \mu \mathrm{L}$ of phosphate buffered saline and immediately transferred to the cellular and molecular oral biology laboratory of Shahid Beheshti University, School of Dentistry. The samples were stored in a freezer at $-70^{\circ} \mathrm{C}$ until DNA extraction. Microbial analysis was carried out for detection of microorganisms. Using Scion Image (USA) software designed for scanning of digital images, the light intensity of bands on electrophoresis gel was converted to numbers. In other words, the white light intensity of each band, indicative of the amount of DNA in that specific band, was converted to pixel numbers in each band (semi-quantitative analysis).

\section{Results}

Porphyromonas gingivalis $(\mathrm{Pg})$ count decreased in the intervention groups at one month post-treatment. At 3 months, its count increased in all groups except for laser + SRP (Figure 1).

Actinobacillus actinomycetemcomitans (Aa) count increased after PDT, but decreased in other groups at 1 month. At 3 months, bacteria were not present in any of the intervention groups, but Aa was still found in the control group. Thus, all the interventions effectively eradicated Aa after 3 months of treatment (Figure 2).

Treponema denticola (Td) count increased in the control group, but decreased in the intervention groups at one month post-intervention. At 3 months post-intervention, Td count increased in the PDT group compared to its value at one month; however, its count at three months was still lower than at baseline (Figure 3).

Fusobacterium nucleatum (Fn) count slightly increased in the control and laser curettage groups at 1 month post-treatment but decreased in PDT and laser + SRP groups compared to the baseline value prior to treatment. At 3 months, Fn count increased compared to its values at baseline and 1 month in all groups (Figure 4).

The greatest reduction in Tannerella forsythensis count (Tf) was noted in the laser curettage and laser + SRP groups. Three months post-treatment, Tf count increased

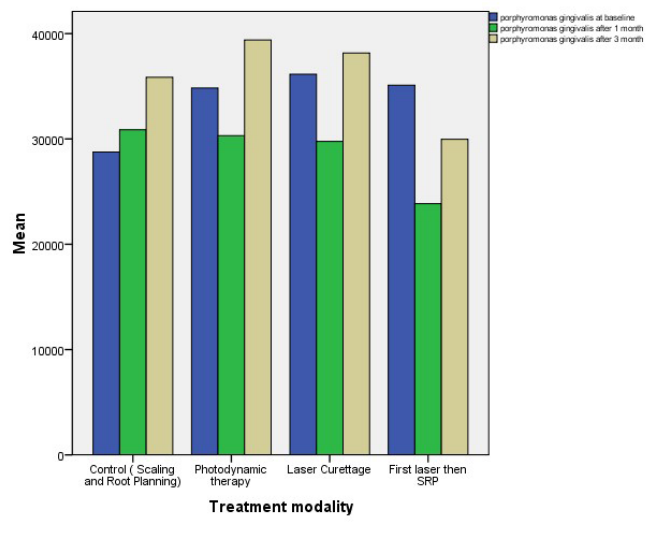

Figure 1. Diagram of Semi-quantitative Analysis of Porphyromonas gingivalis. 


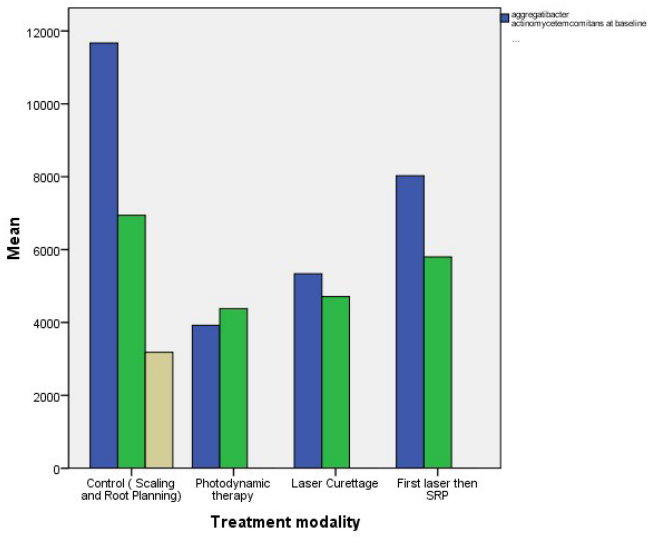

Figure 2. Diagram of Semi-quantitative Analysis of Actinobacillus actinomycetemcomitans.

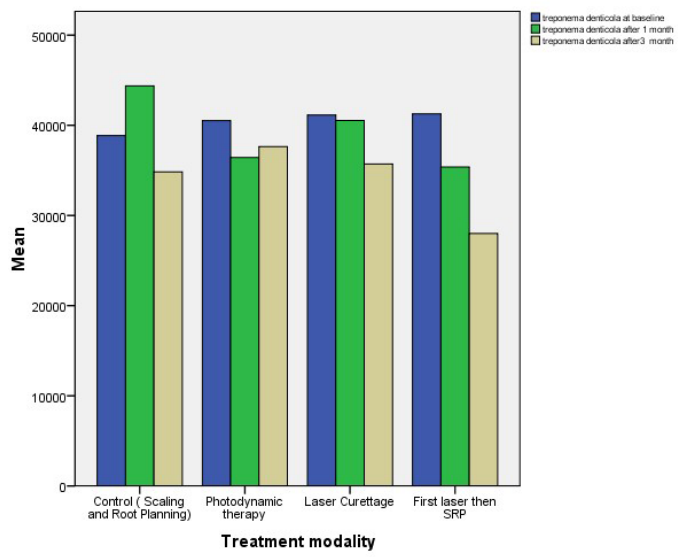

Figure 3. Diagram of Semi-quantitative analysis of Treponema denticola.

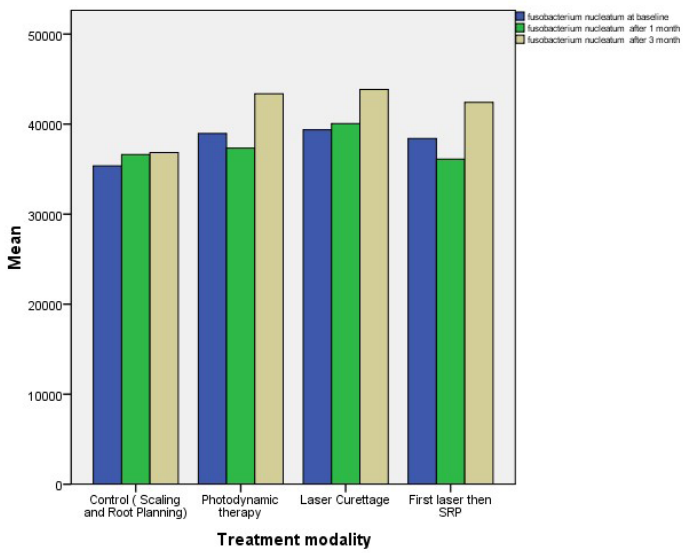

Figure 4. Diagram of Semi-quantitative Analysis of Fusobacterium nucleatum.

in the control and all intervention groups except for laser + SRP group compared to its values at 1 month. At 3 months post-treatment, the Tf count was still lower than its baseline value in all groups (Figure 5).

Prevotella intermedia $(\mathrm{Pi})$ increased in the control and intervention groups except for laser + SRP group at 1 month post-treatment. At 3 months, this value increased in all

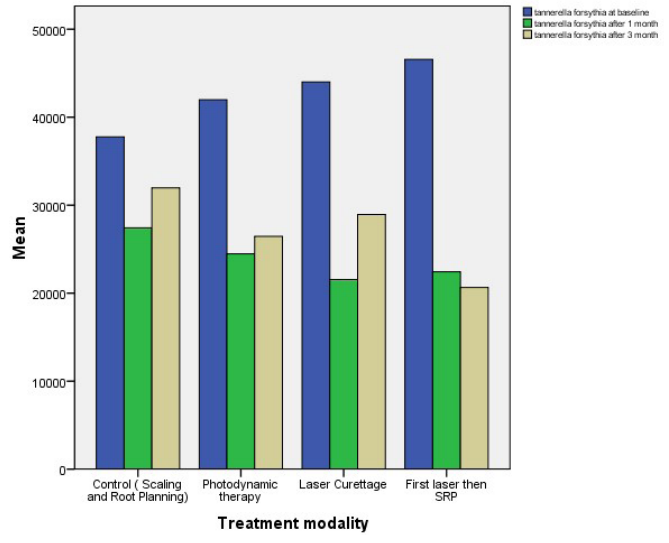

Figure 5. Diagram of Semi-quantitative Analysis of Tannerella forsythensis Count.

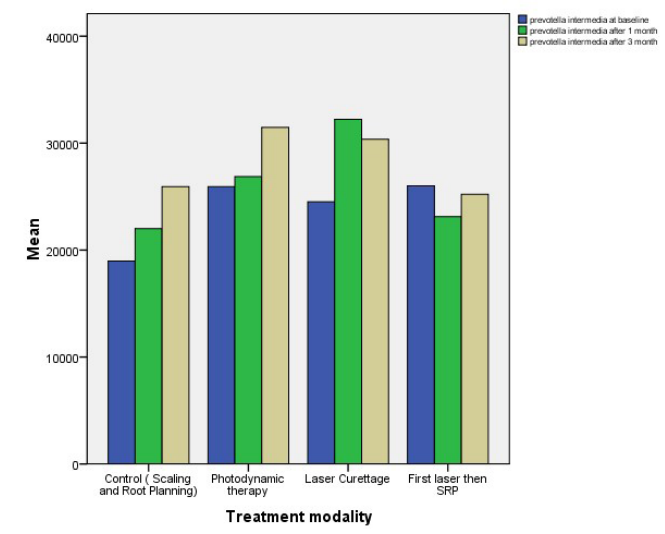

Figure 6. Diagram of Semi-quantitative Analysis of Prevotella intermedia.

groups except for laser curettage group (Figure 6).

\section{Discussion}

Mechanical debridement can significantly change the microbiological environment of periodontal pockets via converting the pathogenic biofilm to beneficial biofilm. Under these circumstances, the microbial loading and bacterial byproducts such as lipopolysaccharides decrease, host immunity and inflammatory reactions are better controlled, the flow of gingival crevicular fluid decreases, and a neutral subgingival area and healthy periodontium achieved. In this study, the efficacy of PDT as an adjunct to non-surgical periodontal therapy was evaluated.

Clinical studies have reported controversial outcomes for PDT and non-surgical periodontal therapy ${ }^{13}$ ), and some researchers have reported significant improvement in clinical parameters following PDT and SRP compared to SRP alone. ${ }^{14,15}$ However, some others reported that the use of PDT as an adjunct had no significant effects. ${ }^{16-18}$ The results of a study by Christodoulides et al in 2008 were somehow in accord with the findings of the current study. ${ }^{15}$ Alwaeli et $\mathrm{al}^{19}$ in 2013 and Berakdar et $\mathrm{al}^{20}$ in 2012 reported that PDT had greater efficacy in decreasing the probing PD 3 months after treatment, which is in line with the current findings. 
De Micheli et al in 2011 showed that laser irradiation along with non-surgical periodontal therapy had no superior efficacy to conventional periodontal therapy ${ }^{21}$; which is in contrast to the results of the current study. They also reported that probing PD was significantly greater in the control compared to the experimental group; however, the two groups were similar in terms of PI and BOP. ${ }^{21}$ No such results were obtained in the current study. Difference in diode laser parameters such as type of laser, photosensitizer, wavelength, density, energy and time duration may be responsible for the controversial results. Moreover, single-session or multiple sessions of PDT have been investigated in previous studies. Lulic et $\mathrm{al}^{22}$ in 2009 demonstrated that multiple sessions of PDT as an adjunct had several clinical advantages for periodontal pockets. Dukić et al in 2013 reported that multiple applications of diode laser along with SRP decreased the probing PD in moderate-depth $(4-6 \mathrm{~mm})$ periodontal pockets. ${ }^{23}$ In the current study, single-session PDT along with SRP was conducted in areas of chronic periodontitis and had significant efficacy in decreasing PD and eliminating pathogenic bacteria compared to SRP alone. Thus, parameters such as laser irradiation settings, contact time, number of treatment sessions and other PDT parameters all affect the outcome of periodontal therapy.

In the current study, qualitative analysis showed that Fn and Td were eradicated one month after different therapeutic modalities. PDT had greater efficacy in decreasing Pi compared to other modalities at one and three months post-treatment. Tf count increased in all four groups. Also, Aa count decreased in all groups and Pg count increased in all groups at 1 and 3 months post-treatment. Non-surgical subgingival debridement significantly decreased the bacterial count related to chronic periodontitis. ${ }^{24,25}$ However, some strains such as Aa and Pg are highly resistant to subgingival debridement. ${ }^{26}$ This is believed to be related to their ability in invading the pocket epithelium and the underlying connective tissue. ${ }^{24,27}$ Presence of pathogenic bacteria in pockets is related to increased pocket depth, continuous bleeding and increased risk of disease progression. ${ }^{28}$ Theodoro et al in 2012 showed that periodontal therapy caused a significant reduction in the count of main periodontal pathogens but their clinical results were not significantly different. ${ }^{29}$ Reduction in percentage of areas that tested positive for Pg and Pi in PDT group was greater than in SRP alone. This finding is not in accord with our results because in our study the frequency of areas that tested positive for $\mathrm{Pi}$ and $\mathrm{Pg}$ increased to some extent at three months post-treatment. Aimetti et al in 2004 showed that Pg and Pi had a significant association with increased resistance to periodontal therapy. These findings justify the use of PDT as an adjunct to conventional periodontal therapy. ${ }^{30}$

\section{Conclusion}

PDT as an adjunct to periodontal therapy appears to be more effective than conventional periodontal therapy alone. However, in terms of reduction in count of some pathogenic strains, this method did not have significant effects in comparison with conventional non-surgical periodontal therapy. However, numerous variables related to the results of previous studies make it difficult to reach a consensus regarding the efficacy of PDT.

\section{Ethical Considerations}

This study have been approved by ethical committee of Shahid Beheshti University of Medical Science.

\section{Conflict of Interest}

We had not any conflict of interests during this project.

\section{References}

1. Armitage GC. Development of a classification system for periodontal diseases and conditions. Ann Periodontol. 1999;4:1-6. doi:10.1902/annals.1999.4.1.1.

2. O'Leary TJ. The impact of research on scaling and root planing. J Periodontol. 1986;57:69-75. doi: 10.1902/ jop.1986.57.2.69.

3. Cugini MA, Haffajee AD, Smith C, Kent RL Jr, Socransky SS. The effect of scaling and root planing on the clinical and microbiological parameters of periodontal diseases: 12-month results. J Clin Periodontol. 2000;27(1):30-36. doi:10.1034/j.1600-051x.2000.027001030.x.

4. Caruso U, Nastri L, Piccolomini R, d'Ercole S, Mazza C, Guida L. Use of diode laser $980 \mathrm{~nm}$ as adjunctive therapy in the treatment of chronic periodontitis. A randomized controlled clinical trial. New Microbiologica. 2008;31:513518.

5. Harris DM, Yessik M. Therapeutic ratio quantifies laser antisepsis: ablation of Porphyromonas gingivalis with dental lasers. Lasers Surg Med. 2004;35(3):206-213. doi:10.1002/lsm.20086.

6. Karlsson MR, Diogo Lofgren CI, Jansson HM. The effect of laser therapy as an adjunct to non-surgicalperiodontal treatment in subjects with chronic periodontitis: a systematic review. J Periodontol. 2008;79(11):2021-2028. doi:10.1902/jop.2008.080197

7. Chan Y, Lai CH. Bactericidal effects of different laser wavelengths on periodonto-pathic germs in photodynamic therapy. Lasers Med Sci 2003;18(1):51-55.

8. O’Neill JF, Hope CK, Wilson M. Oral bacteria inmultispecies biofilms can be killed by red light inthe presence of toluidine blue. Lasers Surg Med. 2002;31(2):86-90. doi:10.1002/lsm.10087.

9. Raab O. The effect of fluorescent agents oninfusoria (in German). Z Biol. 1900;39:524-526.

10. Raghavendra M, Koregol A, Bhola S. Photodynamic therapy: a targeted therapy in periodontics. Aust Dent J. 2009;54(1):102-109. doi:10.1111/j.18347819.2009.01148.x.

11. Hope $\mathrm{C}$, Wilson M. Induction of lethal photosensitization in biofilms using a confocal scanning laser as the excitation source. J Antimicrob Chemother. 2006;57(6):1227-1230. doi:10.1093/jac/dkl096.

12. Garcez A, Ribeiro M, Tegos G, Nunez S, Jorge A, Hamblin M. Antimicrobial photodynamic therapy combined with conventional endodontic treatment to eliminate root canal biofilm infection. Lasers Surg Med. 2007;39(1):59-66. doi:10.1002/lsm.20415.

13. Braun A, Dehn C, Krause F, Jepsen S. Short-term clinical 
effects of adjunctive antimicrobial photodynamic therapy in periodontal treatment: a randomized clinical trial. $J$ Clin Periodontol. 2008;35(10):877-884. doi:10.1111/j.1600051x.2008.01303.x.

14. Andersen R, Loebel N, Hammond D, Wilson M. Treatment of periodontal disease by photo-disinfection compared to scaling and root planning. J Clin Dent. 2007;18(2):34-38.

15. Christodoulides N, Nikolidakis D, Chondros P, et al. Photodynamic therapy as an adjunct to non-surgical periodontal treatment: a randomized, controlled clinical trial. J Periodontol. 2008;79(9):1638-1644. doi:10.1902/ jop.2008.070652.

16. Yilmaz S, Kuru B, Kuru L, Noyan U, Argun D, Kadir T. Effect of gallium arsenide diode laser on human periodontal disease: a microbiological and clinical study. Lasers Surg Med. 2002;30(1):60-66. doi:10.1002/lsm.10010.

17. de Oliveira RR, Schwartz-Filho HO, Novaes AB. Antimicrobial photodynamic therapy in the non-surgical treatment of aggressive periodontitis: cytokine profile in gingival crevicular fluid, preliminary results. J Periodontol. 2009;80:98-105. doi:10.1902/jop.2009.070465.

18. Polansky R, Haas M, Heschl A, Wimmer G. Clinical effectiveness of photodynamic therapy in the treatment of periodontitis. J Clin Periodontol. 2009;36(7):575-580. doi:10.1111/j.1600-051x.2009.01412.x.

19. Alwaeli HA, Al-Khateeb SN, Al-Sadi A. Long-term clinical effect of adjunctive antimicrobial photodynamic therapy in periodontal treatment: a randomized clinical trial. Lasers Med Sci. 2015;30(2):801-807. doi:10.1007/s10103-0131426-y.

20. Berakdar M, Callaway A, Fakhr Eddin M, Rob A, Willershausen B. Comparison between scaling-root planing (srp) and srp/photodynamic therapy: six-months study. Head Face Med. 2012;8:12. doi:10.1186/1746160x-8-12.

21. De Micheli G, de Andrade AK, Euzebio Alves VT, Seto M, Pannuti CM, Cai S. Efficacy of high intensity diode laser as an adjunct to non-surgical periodontal treatment: a randomized controlled trial. Lasers Med Sci. 2011;26:43-48. doi:10.1007/s10103-009-0753-5.

22. Lulic M, Leiggener Görög I, Salvi GE, Ramseier CA, Mattheos N, Lang NP. One-year outcomes of repeated adjunctive photodynamic therapy during periodontal maintenance: a proof of principle randomized-controlled clinical trial. J Clin Periodontol. 2009;36(8):661-666.

23. Dukić W, Bago I, Aurer A, Roguljić M. Clinical effectiveness of diode laser therapy as an adjunct to non-surgical periodontal treatment: a randomized clinical study. $J$ Periodontol. 2013;84(8):1111-1117.

24. Shiloah J, Patters MR. DNA probe analyses of the survival of selected periodontal pathogens following scaling, root planing, and intra-pocket irrigation. J Periodontol. 1994;65(6):568-575. doi:10.1902/jop.1994.65.6.568.

25. Darby IB, Hodge PJ, Riggio MP, Kinane DF. Clinical and microbiological effect of scaling and root planing in smoker and non-smoker chronic and aggressive periodontitis patients. J Clin Periodontol. 2005;32(2):200206. doi:10.1111/j.1600-051x.2005.00644.x.

26. Haffajee AD, Teles RP, Socransky SS. The effect of periodontal therapy on the composition of the subgingival microbiota. Periodontology 2000. 2006;42(1):219-258. doi:10.1111/j.1600-0757.2006.00191.x.

27. Renvert S, Wikstrom M, Dahlen G, Slots J, Egelberg J. On the inability of root debridement and periodontal surgery to eliminate Actinobacillus actinomycetemcomitans from periodontal pockets. J Clin Periodontol. 1990;17(6):351355. doi:10.1111/j.1600-051x.1990.tb00030.x.

28. Mombelli A, Schmid B, Rutar A, Lang NP. Persistence patterns of Porphyromonas gingivalis, Prevotella intermedia/nigrescens, and Actinobacillus actinomyetemcomitans after mechanical therapy of periodontal disease. J Periodontol. 2000;71(1):14-21. doi:10.1111/j.1600-051x.1990.tb00030.x.

29. Theodoro LH, Silva SP, Pires JR, et al. Clinical and microbiological effects of photodynamic therapy associated with nonsurgical periodontal treatment: a 6-month followup. Lasers Med Sci. 2012;27(4):687-693. doi:10.1007/ s10103-011-0942-x.

30. Aimetti M, Romano F, Torta I, Caposio P, Romagnoli R. Debridement and local application of tetracycline-loaded fibres in the management of persistent periodontitis: results after 12 months. J Clin Periodontol. 2004;31(3):166172. doi:10.1111/j.0303-6979.2004.00457.x. 